\title{
Laplace-Beltrami equation on a hypersurface with Lipschitz boundary
}

\section{L'équation de Laplace-Beltrami sur une hypersurface avec un bord de Lipschitz}

\section{R. Duduchava}

The University of Georgia, M. Kostava str. 77A, Tbilisi 0171, Georgia

r.duduchava@ug.edu.ge

A. Razmadze Mathematical Institute, I. Javakhishvili Tbilisi State University,

M. Tamarashvili str. 6, Tbilisi 0177, Georgia

roland.duduchava@tsu.ge

ABSTRACT. Main objective of the present paper is to prove solvability of the Dirichlet, Neumann and Mixed boundary value problems for an anisotropic Laplace-Beltrami equation on a hypersurface $\mathcal{C}$ with the Lipschitz boundary $\Gamma=\partial \mathcal{C}$ in the classical $\mathbb{W}^{1}(\mathcal{C})$ space setting.

2010 Mathematics Subject Classification: 35J57, 58J32.

KEYWORDS. Hypersurface with Lipschitz boundary; Anisotropic Laplace Beltrami equation; Dirichlet BVP; Neumann BVP; Mixed type BVP; Günter's derivatives; Lax-Milgram lemma; Bessel potential spaces.

\section{Introduction}

Let $\mathcal{C}$ be a sufficiently smooth hypersurface in the Euclidean space $\mathbb{R}^{n}$ with Lipschitz boundary $\Gamma:=\partial \mathcal{C}$ (see Section 4 for definitions).

In the present paper we investigate Basic Boundary Value Problems (Basic BVPs) for the "anisotropic" Laplace-Beltrami equation

$$
\operatorname{div}_{\mathcal{C}} \mathcal{A} \nabla_{\mathcal{C}} \varphi(\mathcal{X})=f(\mathcal{X}), \quad \mathcal{X} \in \mathcal{C} .
$$

Here $\operatorname{div}_{\mathcal{C}} \boldsymbol{V}=\mathcal{D}_{1} V_{1}+\cdots+\mathcal{D}_{n} V_{n}, \boldsymbol{V}=\left(V_{1}, \ldots, V_{n}\right)^{\top}$ and $\boldsymbol{\nabla}_{\mathcal{C}} \varphi=\left(\mathcal{D}_{1} \varphi, \ldots, \mathcal{D}_{n} \varphi\right)^{\top}$ are the surface divergence and surface gradient on $\mathcal{C}$ and $\mathcal{D}_{1}, \ldots, \mathcal{D}_{n}$ are the Günter's tangent derivatives (see Section 4 for details). $\mathcal{A}$ is a positive definite $n \times n$ matrix function

$$
\langle\mathcal{A}(\mathcal{X}) \xi, \xi\rangle \geqslant C|\xi|^{2}>0 \quad \text { for all } \xi=\left(\xi_{1}, \ldots, \xi_{n}\right)^{\top} \in \mathbb{R}^{n},
$$

where $\langle\eta, \xi\rangle:=\sum_{j=1}^{n} \eta_{j} \xi_{j}$ denotes the scalar product in $\mathbb{R}^{n}$.

If $\mathcal{A}$ is the identity matrix, the "anisotropic" Laplace-Beltrami equation (1.1) transforms into the classical Laplace-Beltrami equation

$$
\Delta_{\mathcal{C}} \varphi(\mathcal{X})=f(\mathcal{X}), \quad \mathcal{X} \in \mathcal{C},
$$

where the well-known Laplace-Beltrami operator on the surface $\Delta_{\mathcal{C}}$, written in the Günter's tangent derivatives, acquires the following simple form

$$
\Delta_{\mathcal{C}} \varphi=\operatorname{div}_{\mathcal{C}} \nabla_{\mathcal{C}} \varphi=\mathcal{D}_{1}^{2} \varphi+\ldots+\mathcal{D}_{n}^{2} \varphi
$$

The operator in equation (1.1) is positive definite and Lax-Milgram Lemma applies to prove solvability of Basic BVPs for such equations in the classical setting $\varphi \in \mathbb{W}^{1}(\mathcal{C})$ (see Section 5). Namely, is proved 
the unique solvability of the Dirichlet and mixed (Dirichlet-Neumann) BVPs, while for the Neumann BVP the right-hand side $f$ and the Neumann condition on the boundary have to satisfy one condition (cf. Theorem 5.1).

For isotropic Laplace-Beltrami equation with Dirichlet and Neumann boundary conditions solvability was proved in [9] with the help of potential method and in [15] for Mixed, Dirichlet and Neumann boundry conditions using Lax-Milgram Lemma.

Moreover, for the Dirichlet (5.2) and Neumann (5.3) BVPs and non-classical setting

$$
\begin{array}{r}
u \in \mathbb{H}_{p}^{s}(\mathcal{C}), \quad f \in \widetilde{\mathbb{H}}_{p, 0}^{s-2}(\mathcal{C}), \quad g \in \mathbb{H}_{p}^{s-\frac{1}{p}}(\Gamma), \quad h \in \mathbb{H}_{p}^{s-1-\frac{1}{p}}(\Gamma), \\
1<p<\infty, \quad \frac{1}{p}<s<1+\frac{1}{p}
\end{array}
$$

in case of a hypersurface with the smooth boundary, the unique solvability holds as well (see [9]).

For the mixed BVP (5.3) in the non-classical setting

$$
\begin{array}{r}
u \in \mathbb{H}_{p}^{s}(\mathcal{C}), \quad f \in \widetilde{\mathbb{H}}_{p, 0}^{s-2}(\mathcal{C}), \quad g \in \mathbb{H}_{p}^{s-\frac{1}{p}}\left(\Gamma_{D}\right), \quad h \in \mathbb{H}_{p}^{s-1-\frac{1}{p}}\left(\Gamma_{N}\right), \quad \Gamma=\Gamma_{D} \cup \Gamma_{N}, \\
1<p<\infty, \quad \frac{1}{p}<s<1+\frac{1}{p},
\end{array}
$$

even for a hypersurface with the smooth boundary and for Dirichlet (5.2) and Neumann (5.3) BVPs for a hypersurface with the Lipshitz boundary and the non-classical setting (1.5), the solvability conditions change dramatically (cf. $[13,14,10])$.

Mixed BVPs for the Laplace equation in domains were investigated by Lax-Milgram Lemma by many authors (see, e.g., [27] and the recent lecture notes online [23] for survey).

The paper is organized as follows: in the first Section 1 we expose auxiliary material about Bessel potential, Sobolev spaces on Euclidean $\mathbb{R}^{n}$ space, on domains in $\mathbb{R}^{n}$ and on hypersurfaces without and with boundary, prove a couple of Lemmata. In Section 2 we expose necessary auxiliary information from the operator theory and prove part of them, including the celebrated Lax-Milgram Lemma, used later in Section 4 to prove the main Theorem 5.1 of the present paper. In Section 3 we expose calculus of Günter's tangent differential operators on a hypersurface, which is the main tool to write and investigate boundary value problems for the anisotropic Laplace-Beltrami equation (1.1). In Section 4 we formulate precisely Dirichlet, Neumann and Mixed type BVPs for an anisotropic Laplace-Beltrami equation (1.1) on a hypersurface $\mathcal{C}$ with the Lipschitz boundary $\Gamma=\partial \mathcal{C}$ in classical $\mathbb{W}^{1}(\mathcal{C})$ space setting. After proving some auxiliary propositions, we prove the main Theorem 5.1 on the solvability of the above mentioned 3 classical BVPs in the classical $\mathbb{W}^{1}(\mathcal{C})$ space setting.

\section{Bessel potential spaces}

$\mathbb{S}\left(\mathbb{R}^{n}\right)$ denotes the Schwartz space of all rapidly decaying functions and $\mathbb{S}^{\prime}\left(\mathbb{R}^{n}\right)$ - the dual space of tempered distributions. Since the Fourier transform and its inverse, defined by

$$
\mathcal{F} \varphi(\xi)=\int_{\mathbb{R}^{n}} e^{i \xi x} \varphi(x) d x \quad \text { and } \quad \mathcal{F}^{-1} \psi(x)=(2 \pi)^{-n} \int_{\mathbb{R}^{n}} e^{-i x \xi} \psi(\xi) d \xi, \quad x, \xi \in \mathbb{R}^{n}
$$


are continuous in both spaces $\mathbb{S}\left(\mathbb{R}^{n}\right)$ and $\mathbb{S}^{\prime}\left(\mathbb{R}^{n}\right)$, the convolution operator

$$
\mathbf{a}(D) \varphi=W_{a}^{0} \varphi:=\mathcal{F}^{-1} a \mathcal{F} \varphi \quad \text { with } \quad a \in \mathbb{S}^{\prime}\left(\mathbb{R}^{n}\right), \quad \varphi \in \mathbb{S}\left(\mathbb{R}^{n}\right)
$$

is a continuous transformation from $\mathbb{S}\left(\mathbb{R}^{n}\right)$ into $\mathbb{S}^{\prime}\left(\mathbb{R}^{n}\right)$ (see $\left.[6,12]\right)$.

The Bessel potential space $\mathbb{H}_{p}^{s}\left(\mathbb{R}^{n}\right),-\infty<s<\infty$ is the space of functions from $\mathbb{S}^{\prime}\left(\mathbb{R}^{n}\right)$ which have finite norm

$$
\left\|u\left|\mathbb{H}_{p}^{s}\left(\mathbb{R}^{n}\right) \|:=\right|\left|\Lambda^{s} u\right| L_{p}\left(\mathbb{R}^{n}\right)||<\infty .\right.
$$

Here $\Lambda^{\theta}=\mathcal{F}^{-1}\left(1+|\xi|^{2}\right)^{\frac{\theta}{2}} \mathcal{F}$ is the Bessel potential operator, which arranges the isometrical isomorphism of the Bessel potential spaces $\Lambda^{\theta}: \mathbb{H}_{p}^{s}\left(\mathbb{R}^{n}\right) \rightarrow \mathbb{H}_{p}^{s-\theta}\left(\mathbb{R}^{n}\right)$ for all $-\infty<s, \theta<\infty$.

For an integer $m=1,2, \ldots$ and $1 \leqslant p \leqslant \infty$, the Bessel potential space $\mathbb{H}_{p}^{s}\left(\mathbb{R}^{n}\right)$ coincides with the Sobolev space $\mathbb{W}_{p}^{m}\left(\mathbb{R}^{n}\right)$ of those functions in $\varphi \in \mathbb{L}_{p}\left(\mathbb{R}^{n}\right)$ which have distributional derivatives $\partial^{\alpha} \varphi$ of order $|\alpha| \leqslant m$ in the same space $\partial^{\alpha} \varphi \in \mathbb{L}_{p}\left(\mathbb{R}^{n}\right)$. The space is endowed with the natural equivalent norm

$$
\left\|f \mid \mathbb{W}_{p}^{m}\left(\mathbb{R}^{n}\right)\right\|:=\left(\sum_{|\alpha| \leqslant m}\left\|\partial^{\alpha} f \mid \mathbb{L}_{p}\left(\mathbb{R}^{n}\right)\right\|^{p}\right)^{1 / p}
$$

for $1 \leqslant p<\infty$, with the usual sup-norm modification for $p=\infty$ :

$$
\left\|f\left|\mathbb{W}_{\infty}^{m}\left(\mathbb{R}^{n}\right) \|:=\sum_{|\alpha| \leqslant m} \sup _{x \in \mathbb{R}^{n}}\right| \partial^{\alpha} f(x) \mid .\right.
$$

Let

$$
\mathbb{R}_{+}^{n}:=\left\{x=\left(x_{1}, \ldots, x_{n-1}, x_{n}\right)^{\top} \in \mathbb{R}^{n}: x_{n}>0\right\}
$$

$\mathbb{R}_{+}^{n}$ denote the half space. The space $\widetilde{\mathbb{H}}_{p}^{\theta}\left(\mathbb{R}_{+}^{n}\right)$ is defined as the subspace of $\mathbb{H}_{p}^{\theta}\left(\mathbb{R}^{n}\right)$ of those functions $\varphi \in \mathbb{H}_{p}^{\theta}\left(\mathbb{R}^{n}\right)$, which are supported in the half space, $\operatorname{supp} \varphi \subset \overline{\mathbb{R}_{+}^{n}}$, whereas $\mathbb{H}_{p}^{\theta}\left(\mathbb{R}_{+}^{n}\right)$ denotes the quotient space $\mathbb{H}_{p}^{\theta}\left(\mathbb{R}_{+}^{n}\right)=\mathbb{H}_{p}^{\theta}\left(\mathbb{R}^{n}\right) / \widetilde{\mathbb{H}}_{p}^{\theta}\left(\mathbb{R}_{-}^{n}\right), \mathbb{R}_{-}^{n}:=\mathbb{R}^{n} \backslash \overline{\mathbb{R}_{+}^{n}}$ and can be identified with the space of distributions $\varphi$ on $\mathbb{R}_{+}^{n}$ which admit extensions up to a function in $\mathbb{H}_{p}^{\theta}\left(\mathbb{R}^{n}\right)$. Therefore $r_{+} \mathbb{H}_{p}^{\theta}\left(\mathbb{R}^{n}\right)=\mathbb{H}_{p}^{\theta}\left(\mathbb{R}_{+}^{n}\right)$, where $r_{+}=r_{\mathbb{R}_{+}^{n}}$ denotes the restriction from $\mathbb{R}^{n}$ to the half space $\mathbb{R}_{+}^{n}$.

The subspace $\widetilde{H}_{p}^{\theta}\left(\mathbb{R}_{+}^{n}\right)$ inherits the norm the of the ambient space $\mathbb{H}_{p}^{\theta}\left(\mathbb{R}^{n}\right)$, while the norm in $\mathbb{H}_{p}^{s}\left(\mathbb{R}^{+}\right)$ is defined by

$$
\| f \mid \mathbb{H}_{p}^{s}\left(\mathbb{R}^{+}\left\|=\inf _{\ell}\right\| \ell f \mid \mathbb{H}_{p}^{s}(\mathbb{R}) \|\right.
$$

where $\ell f$ stands for any extension of $f$ to a distribution in $\mathbb{H}_{p}^{s}(\mathbb{R})$.

Note that $\widetilde{\mathbb{H}}_{p}^{s}\left(\mathbb{R}^{+}\right)$is always continuously embedded in $\mathbb{H}_{p}^{s}\left(\mathbb{R}^{+}\right)$and for $s \in(1 / p-1,1 / p)$ these two spaces coincide.

The spaces $\mathbb{H}_{p}^{\theta}(\Omega), \widetilde{\mathbb{H}}_{p}^{\theta}(\Omega), \mathbb{H}_{p}^{\theta}(\mathcal{M})$ and $\widetilde{\mathbb{H}} \theta_{p}^{\theta}(\mathcal{M})$ on a domain $\Omega \subset \mathbb{R}^{n}$ with the boundary $\partial \Omega \neq \emptyset$ and on a smooth manifold (on a hypersurface in particular) $\mathcal{M}$ with the boundary $\partial \mathcal{M} \neq \emptyset$ are defined in a standard way using a coordinate diffeomorphisms and the partition of the unity (see [29, 30] for details). 
Let $\mathcal{M}$ be a compact, smooth closed or open hypersurfacea with a non-trivial measure mes $\mathcal{M} \neq 0$ and $s \in \mathbb{R}, 1<p<\infty$. If $\mathcal{M}$ is definitely closed, we use $\mathcal{S}$, while in case $\mathcal{M}$ is definitely open, we use $\mathcal{C}$.

By $\mathbb{X}_{p}^{s}(\mathcal{M})$ we denote one of the spaces: $\mathbb{H}_{p}^{s}(\mathcal{M})$, Sobolev-Slobodecki $\mathbb{W}_{p}^{s}(\mathcal{M})$ (if $\mathcal{M}$ is closed or open) and by $\widetilde{\mathbb{X}}_{p}^{s}(\mathcal{C})$ denote one of the spaces: $\widetilde{\mathbb{H}}_{p}^{s}(\mathcal{C})$ and $\widetilde{\mathbb{W}}_{p}^{s}(\mathcal{C})$. Consider the space

$$
\mathbb{X}_{p, \#}^{s}(\mathcal{M}):=\left\{\varphi \in \mathbb{X}_{p}^{s}(\mathcal{M}):(\varphi, 1)=0\right\}
$$

where $(\varphi, \psi):=\int_{\mathcal{M}} \varphi(t) \overline{\psi(t)} d t$ denotes the scalar product in $\mathbb{L}_{2}(\mathcal{M})$ for $s \geqslant 0$, while for $s<0$ is interpreted as the functional $\psi \in \mathbb{X}_{p^{\prime}}^{-s}(\mathcal{M})$ applied to $\varphi \in \mathbb{X}_{p}^{s}(\mathcal{M})$. Note, that $(\varphi, 1)$ exists if $\varphi \in \mathbb{X}_{p}^{s}(\mathcal{M})$ for $s \geqslant 0$ because $\mathbb{X}_{p}^{s}(\mathcal{M}) \subset \mathbb{L}_{1}(\mathcal{M})$ and for $s<0$ because $1 \in \mathbb{X}_{p^{\prime}}^{-s}(\mathcal{M})$.

It is obvious that $\mathbb{X}_{p, \#}^{s}(\mathcal{M})$ does not contain constants: if $c_{0}=$ const $\in \mathbb{X}_{p, \#}^{s}(\mathcal{M})$, then

$$
0=\left(c_{0}, 1\right)=c_{0}(1,1)=c_{0} \operatorname{mes} \mathcal{M}
$$

and $c_{0}=0$. Moreover, $\mathbb{X}_{p}^{s}(\mathcal{M})$ decomposes into the direct sum

$$
\mathbb{X}_{p}^{s}(\mathcal{M})=\mathbb{X}_{p, \#}^{s}(\mathcal{M})+\{\text { const }\}
$$

and the dual (adjoint) space is

$$
\left.\left(\mathbb{X}_{p, \#}^{s, \mathcal{M}}\right)\right)^{*}=\mathbb{X}_{p^{\prime}, \#}^{-s}(\mathcal{M}), \quad p^{\prime}:=\frac{p}{p-1}
$$

Indeed, decomposition (2.4) follows from the representation

$$
\varphi=\varphi_{0}+\varphi_{\text {aver }}, \quad \varphi_{0} \in \mathbb{X}_{p, \#}^{s}(\mathcal{M}), \quad \varphi_{\text {aver }}:=\frac{1}{\operatorname{mes} \mathcal{M}}(\varphi, 1)
$$

of arbitrary function $\varphi \in \mathbb{X}_{p}^{s}(\mathcal{M})$, because $\left(\varphi_{0}\right)_{\text {aver }}=\left(\varphi-\varphi_{\text {aver }}\right)_{\text {aver }}=0$.

Description (2.5) of the dual space follows from the fact that the dual space to $\mathbb{X}_{p}^{s}(\mathcal{M})$ is $\mathbb{X}_{p^{\prime}}^{-s}(\mathcal{M})$ (see [30]) and, therefore, due to the decomposition (2.4) and Hahn-Banach theorem, the dual space to $\mathbb{X}_{p, \#}^{s}(\mathcal{M})$ should be embedded into $\mathbb{X}_{p^{\prime}}^{-s}(\mathcal{M})$. The only functional from $\mathbb{X}_{p^{\prime}}^{-s}(\mathcal{M})$ that vanishes on the entire space $\mathbb{X}_{p, \#}^{s}(\mathcal{M})$ is constant $1 \in \mathbb{X}_{p^{\prime}}^{-s}(\mathcal{M})$ (see definition (2.3)). After detaching this functional the remainder coincides, due to (2.4), with the space $\mathbb{X}_{p^{\prime}, \#}^{-s}(\mathcal{M})$, which is the dual to $\mathbb{X}_{p, \#}^{s}(\mathcal{M})$.

Let $\Gamma_{0} \subset \Gamma$ be a non-trivial subset of the boundary $\Gamma=\partial \mathcal{C}$ of the surface $\mathcal{C}$ and $\widetilde{\mathbb{X}}_{p}^{s}\left(\Gamma_{0}, \mathcal{C}\right), s>1 / p$, $1<p<\infty$, denote the subspace of $\mathbb{X}_{p}^{s}(\mathcal{C})$ which consists of functions with vanishing trace on $\Gamma_{0}$.

The equivalent norm in the space $\mathbb{W}_{p, \#}^{1}(\mathcal{M})$ is defined as follows

$$
\left\|\varphi\left|\mathbb{W}_{p, \#}^{1}(\mathcal{M})\left\|_{0}:=\right\| \nabla_{\mathcal{S}} \varphi\right| \mathbb{L}_{p}(\mathcal{M})\right\|
$$

and the equivalent norm in the space $\widetilde{\mathbb{W}}_{p}^{1}\left(\Gamma_{0}, \mathcal{C}\right)$ is defined as follows

$$
\left\|\varphi\left|\widetilde{\mathbb{W}}_{p}^{1}\left(\Gamma_{0}, \mathcal{C}\right)\left\|_{0}:=\right\| \nabla_{\mathcal{S}} \varphi\right| \mathbb{L}_{p}(\mathcal{C})\right\|
$$

Indeed, the proofs in the both cases are similar and well known. Therefore we will sketch the proof of only (2.6) for the readers convenience.

Note first that (2.6) defines a norm indeed, since from $\varphi \in \mathbb{W}_{p, \#}^{1},\left\|\nabla_{\mathcal{S}} \varphi \mid \mathbb{L}_{p}(\mathcal{M})\right\|=0$ follows $\nabla_{\mathcal{S}} \varphi=$ 0 and, therefore, $\varphi=$ const (cf. Corollary 4.4 below). Then $\varphi=0$ due to the representations (2.4). 
On the other hand, $\mathbb{W}_{p, \#}^{1}(\mathcal{M})$ is a subspace of $\mathbb{W}_{p}^{1}(\mathcal{M})$ with the induced norm

$$
\left\|\varphi\left|\mathbb{W}_{p, \#}^{1}(\mathcal{M})\left\|:=\sum_{0 \leqslant|\alpha| \leqslant 1}\right\| \mathcal{D}^{\alpha} \varphi\right| \mathbb{L}_{p}(\mathcal{M})\right\| .
$$

From (2.6) and (2.8) follows the trivial inequality

$$
\left\|\varphi\left|\mathbb{W}_{p, \#}^{1}(\mathcal{M})\left\|_{0} \leqslant\right\| \varphi\right| \mathbb{W}_{p, \#}^{1}(\mathcal{M})\right\|
$$

which ensures the continuous embedding of the space $\mathbb{W}_{p, \#}^{1}(\mathcal{M})$ with the norm $(2.8)$ into the same space with the norm (2.8). Due to the Banach theorem the continuity of the inverse embedding follows and ensures the inverse inequality between norms

$$
\left\|\varphi\left|\mathbb{W}_{p, \#}^{m, 0}(\mathcal{M})\left\|_{0} \leqslant C\right\| \varphi\right| \mathbb{W}_{p, \#}^{m}(\mathcal{M})\right\|
$$

with some constant $C$, which is independent of a function $\varphi$. Equivalence of the norms in (2.6) and in (2.8) is proved.

\section{Auxiliary from the operator theory and Lax-Milgram Lemma}

Lemma 3.1. Let $\mathfrak{B}$ be a Banach space. A linear bounded operator Let $\boldsymbol{A} \in \mathcal{L}(\mathfrak{B})$ satisfies the inequality

$$
\|\boldsymbol{A} \varphi|\mathfrak{B}\|\geqslant C\| \varphi| \mathfrak{B}\|
$$

with some constant $C>0$ if and only if the operator $\boldsymbol{A}$ is injective, $\operatorname{Ker} \boldsymbol{A}=\{0\}$ and is normally solvable, i.e., has the closed image $\operatorname{Im} A=\overline{\operatorname{Im} A}$.

Proof: If inequality (3.1) holds, then $\boldsymbol{A} \varphi=0, \varphi \in \mathfrak{B}$, implies $\varphi=0$ and Ker $\boldsymbol{A}=\{0\}$. Now let $\psi_{j}=\boldsymbol{A} \varphi_{j} \rightarrow \psi_{0}$ (convergence in the norm). The inequality (3.1) implies the convergence $\varphi_{j} \rightarrow \varphi_{0}$. Due to continuity of $\boldsymbol{A}$ this implies $\boldsymbol{A} \varphi_{0}=\psi_{0} \in \operatorname{Im} \boldsymbol{A}$ and the image $\operatorname{Im} \boldsymbol{A}$ is closed.

Vice versa, let $\boldsymbol{A}$ be normally solvable and $\operatorname{Ker} \boldsymbol{A}=\{0\}$. Then $\operatorname{Im} \boldsymbol{A}$ is a Hilbert space, subspace of $\mathfrak{B}$ and the operator $\boldsymbol{A}: \mathfrak{B} \rightarrow \operatorname{Im} \boldsymbol{A}$ is bijective. Due to the Banach Inverse mapping theorem, $\boldsymbol{A}$ is invertible: there exists $\mathbf{B} \in \mathcal{L}(\operatorname{Im} \boldsymbol{A}, \mathfrak{B})$ such that $\mathbf{A B} x=x$ and $\mathbf{B A} y=y$ for all $x \in \operatorname{Im} \boldsymbol{A}$ and all $y \in \mathfrak{B}$. Inserting in $\|\mathbf{B} \psi|\mathfrak{B}\|\leqslant C\| \psi| \operatorname{Im} \boldsymbol{A}\|:=\|\psi \mid \mathfrak{B}\|$ the equality $\psi=\boldsymbol{A} \varphi, \varphi \in \mathfrak{B}$, we get (3.1).

Further the present section we assume that $\mathfrak{H}$ is a Hilbert space with respect to some continuous scalar product, a bilinear form $(\cdot, \cdot): \mathfrak{H} \times \mathfrak{H} \rightarrow \mathbb{C}$. Recall that the dual operator $\left(\boldsymbol{A}^{*} \varphi, \psi\right)=(\varphi, \boldsymbol{A} \psi)$ maps continuously the same space $\boldsymbol{A}^{*}: \mathfrak{H} \rightarrow \mathfrak{H}$ and $\boldsymbol{A} \in \mathcal{L}(\mathfrak{H})$ is self-adjoint operator if

$$
(\boldsymbol{A} \varphi, \psi)=(\varphi, \boldsymbol{A} \psi), \quad \forall \varphi, \psi \in \mathfrak{H} .
$$

$\boldsymbol{A} \in \mathcal{L}(\mathfrak{H}, \mathfrak{H})$ is positive definite (or coercive) if the inequality

$$
(\boldsymbol{A} \varphi, \varphi) \geqslant C\|\varphi \mid \mathfrak{H}\|^{2}
$$

holds for some constant $C>0$ and all $\varphi \in \mathfrak{H}$.

Definition 3.2. For an operator $\boldsymbol{A} \in \mathcal{L}(\mathfrak{H})$ the closed set

$$
\Sigma(\boldsymbol{A}):=\overline{\{(\boldsymbol{A} \varphi, \varphi): \varphi \in \mathfrak{H}\}},
$$

where the overbar denotes closing of the set, is called the spectral set of $\boldsymbol{A}$. 
Lemma 3.3 (cf. [19], §V.5.2). If the spectral set $\Sigma(\boldsymbol{A})$ of an operator $\boldsymbol{A} \in \mathcal{L}(\mathfrak{H})$ is real-valued $\Sigma(\boldsymbol{A}) \subset \mathbb{R}$, then $\boldsymbol{A}$ is self-adjoint.

Corollary 3.4. If an operator $\boldsymbol{A} \in \mathcal{L}(\mathfrak{H})$ is positive definite, it is self-adjoint and invertible.

Proof: If $\boldsymbol{A}$ is positive definite, its spectral set is real-valued and $\boldsymbol{A}$ is self-adjoint.

From the boundedness of $\boldsymbol{A}$ and the coerciveness inequality (3.3) we get

$$
\|\boldsymbol{A} \varphi|\mathfrak{H}\|\| \varphi| \mathfrak{H}\| \geqslant(\boldsymbol{A} \varphi, \varphi) \geqslant C\|\varphi \mid \mathfrak{H}\|^{2}
$$

and, further,

$$
\|\boldsymbol{A} \varphi|\mathfrak{H}\|\| \geqslant C\|\varphi \mid \mathfrak{H}\|, \quad \forall \varphi \in \mathfrak{H} .
$$

Due to Lemma 3.1, inequality (3.5) implies that $\boldsymbol{A}$ is normally solvable and has a trivial kernel Ker $\boldsymbol{A}=\{0\}$. Being self-adjoint $\boldsymbol{A}^{*}=\boldsymbol{A}$, the operator has the trivial cokernel as well $\operatorname{dim}$ Coker $\boldsymbol{A}=\operatorname{dim} \operatorname{Ker} \boldsymbol{A}=0$. Therefore, $\boldsymbol{A}$ is invertible.

Let $\mathfrak{H}$ be a Hilbert space and consider a linear Variational problem

$$
a(\varphi, \psi)=L(\psi) \quad \forall \psi \in \mathfrak{H}
$$

where $\varphi \in \mathfrak{H}$ is unknown and

$$
a(\cdot, \cdot): \mathfrak{H} \times \mathfrak{H} \rightarrow \mathbb{R}, \quad L(\cdot): \mathfrak{H} \rightarrow \mathbb{R}
$$

are, respectively, a continuous bilinear form and a continuous linear form (a functional) on $\mathfrak{H}$.

Definition 3.5. We say the Variational problem (3.4) is well-posed if, and only if, for all $\psi \in \mathfrak{H}$, it has one and only one solution $\varphi \in \mathfrak{H}^{*}$, with continuous dependence $\left\|\varphi\left|\mathfrak{H}^{*}\|\leqslant M\| \psi\right| \mathfrak{H}\right\|$ for some constant $M>0$.

We expose the proof of the next assertion, because it not well known.

Let Coker $A_{\mathfrak{B}_{k} \rightarrow \mathfrak{D}_{k}}$ Coker $A_{\mathfrak{B}_{k} \rightarrow \mathfrak{D}_{k}}$ denote a direct complement to the image $\Im A_{\mathfrak{B}_{k} \rightarrow \mathfrak{D}_{k}}$, which is not unique in general.

\section{Theorem 3.6. Let}

$$
A \in \mathcal{F}\left(\mathfrak{B}_{1}, \mathfrak{D}_{1}\right) \cap \mathcal{F}\left(\mathfrak{B}_{2}, \mathfrak{D}_{2}\right),
$$

where $\mathfrak{B}_{1}, \mathfrak{D}_{1}, \mathfrak{B}_{2}$ and $\mathfrak{D}_{2}$ are Banach spaces and the first embedding

$$
\mathfrak{B}_{1} \subset \mathfrak{B}_{2}, \quad \mathfrak{D}_{1} \subset \mathfrak{D}_{2}
$$

holds, while the second embedding is dense. If the indices of $A$ in both pairs of spaces coincide

$$
\text { Ind } A_{\mathfrak{B}_{1} \rightarrow \mathfrak{D}_{1}}=\text { Ind } A_{\mathfrak{B}_{2} \rightarrow \mathfrak{D}_{2}} \text {, }
$$

then the corresponding kernels and the cokernels coincide as well:

$$
\operatorname{Ker} A_{\mathfrak{B}_{1} \rightarrow \mathfrak{D}_{1}}=\operatorname{Ker} A_{\mathfrak{B}_{2} \rightarrow \mathfrak{D}_{2}}
$$

Coker $A_{\mathfrak{B}_{1} \rightarrow \mathfrak{D}_{1}}=$ Coker $A_{\mathfrak{B}_{2} \rightarrow \mathfrak{D}_{2}}$. 
Proof: Due to the first embedding in (3.8)

$\alpha_{1} \leqslant \alpha_{2}, \quad$ where $\quad \alpha_{k}:=\operatorname{dim} \operatorname{Ker} A_{\mathfrak{B}_{k} \rightarrow \mathfrak{D}_{k}}$.

Since

$\operatorname{dim}$ Coker $A_{\mathfrak{B}_{k} \rightarrow \mathfrak{D}_{k}}=\operatorname{dim} \operatorname{Ker} A_{\mathfrak{D}_{k}^{*} \rightarrow \mathfrak{B}_{k}^{*}}^{*}$,

the density of the second embedding in (3.8) yields $\mathfrak{D}_{2}^{*} \subset \mathfrak{D}_{1}^{*}$. Indeed, any functional $F \in \mathfrak{D}_{2}^{*}$ is automatically included in $\mathfrak{D}_{1}^{*}$ : we get $\left|(F, u) \leqslant\|F\|\left\|u\left|\mathfrak{D}_{2}\|\leqslant C\| F\|\| u\right| \mathfrak{D}_{1}\right\|\right.$ and, therefore, $F \in \mathfrak{D}_{1}^{*}$.

On the other hand, any non-trivial functional $F \in \mathfrak{D}_{2}^{*}, F \neq 0$, restricted to $\mathfrak{D}_{1}$, does not vanish $\left.F\right|_{\mathfrak{D}_{1}} \neq 0$. Otherwise, the dense embedding $\mathfrak{D}_{1} \subset \mathfrak{D}_{2}$ implies $F=0$. This completes the proof that the embedding $\mathfrak{D}_{2}^{*} \subset \mathfrak{D}_{1}^{*}$ holds.

Analogously to (3.11) we get

$$
\beta_{2} \leqslant \beta_{1}, \quad \text { where } \quad \beta_{k}:=\operatorname{dim} \text { Coker } A_{\mathfrak{B}_{k} \rightarrow \mathfrak{D}_{k}} .
$$

From (3.11), (3.12) and (3.9), which can yet be written as follows

$$
\alpha_{1}-\beta_{1}=\alpha_{2}-\beta_{2}
$$

we obtain

$$
0 \geqslant \beta_{1}-\beta_{2}=\alpha_{1}-\alpha_{2} \leqslant 0
$$

The latter relations show that $\alpha_{1}=\alpha_{2}, \beta_{1}=\beta_{2}$. These equalities and embedding (3.8) entail (3.10).

Next, we expose the simple but very powerful Lax-Milgram Lemma, but only part of it, which is sufficient for our purposes (the second part deals with the minimization problem of the non-linear functional $\left.\frac{1}{2} a(\varphi, \varphi)-L(\varphi)\right)$.

Lemma 3.7 (Lax-Milgram). Let a continuous bilinear form $a(\cdot, \cdot): \mathfrak{H} \times \mathfrak{H} \rightarrow \mathbb{R}$ in be coercive (cf. (3.3)).

Then the Variational problem (3.4), is well posed: has a unique solution $\varphi \in \mathfrak{B}$ for all $\psi \in \mathfrak{B}$.

For the proof see [22] or, e.g., [27, §1.2.3, Theorem 1.5].

\section{Partial differential operators on hypersurfaces}

Let $\mathcal{S}$ be a hypersurface given by a collection of charts $\left\{\left(\mathcal{S}_{j}, \Theta_{j}\right)\right\}_{j=1}^{M}$, where

$$
\Theta_{j}: \omega_{j} \rightarrow \mathcal{S}_{j}, \quad \mathcal{S}=\bigcup_{j=1}^{M} \mathcal{S}_{j}, \quad \omega_{j} \subset \mathbb{R}^{n-1}, \quad j=1, \ldots, M
$$

The corresponding differentials

$$
D \Theta_{j}(p):=\operatorname{matr}\left[\partial_{1} \Theta_{j}(p), \ldots, \partial_{n-1} \Theta_{j}(p)\right]
$$

have the full $\operatorname{rank} \operatorname{rank} D \Theta_{j}(p)=n-1, \quad \forall p \in \Omega, \quad j=1, \ldots, M$. 
The derivatives

$$
\boldsymbol{g}_{k}=\partial_{k} \Theta_{j}, \quad k=1, \ldots, n-1,
$$

are tangent vector fields on $\mathcal{S}_{j}$ and this system is a basis in the space of tangent vector fields to the surface. The symmetric Gram matrix

$$
\begin{array}{r}
G_{\mathcal{S}}(x):=\left[\left\langle\boldsymbol{g}_{k}(\mathcal{X}), \boldsymbol{g}_{m}(\mathcal{X})\right\rangle\right]_{n-1 \times n-1}=\left[\left\langle\partial_{k} \Theta_{j}(x), \partial_{m} \Theta_{j}(x)\right\rangle\right]_{n-1 \times n-1}, \\
x \in \omega_{j} \subset \mathbb{R}^{n-1}, \quad, j=1, \ldots, M,
\end{array}
$$

defines the natural metric on the space of tangent vector fields on the surface.

The unit normal vector field to the surface $\mathcal{S}$, also known as the Gauß mapping, is defined by the vector product of the covariant basis

$$
\boldsymbol{\nu}(\mathcal{X}):= \pm \frac{\boldsymbol{g}_{1}(\mathcal{X}) \wedge \cdots \wedge \boldsymbol{g}_{n-1}(\mathcal{X})}{\left|\boldsymbol{g}_{1}(\mathcal{X}) \wedge \cdots \wedge \boldsymbol{g}_{n-1}(\mathcal{X})\right|}, \quad \mathcal{X} \in \mathcal{S}
$$

The choice of sign in this formula determines the orientation of the hypersurface. In what follows, we will choose the orientation corresponding to the plus sign in (4.5).

The system of tangent vectors $\left\{\boldsymbol{g}_{k}\right\}_{k=1}^{n-1}$ to $\mathcal{S}$ (cf. (4.3)) is known as the covariant basis. The system of the vector fields

$$
\boldsymbol{g}^{k}=\frac{1}{\operatorname{det} G_{\mathcal{S}}} \boldsymbol{g}_{1} \wedge \cdots \wedge \boldsymbol{g}_{k-1} \wedge \boldsymbol{\nu} \wedge \boldsymbol{g}_{k+1} \wedge \cdots \wedge \boldsymbol{g}_{n-1}, \quad k=1, \ldots, n-1,
$$

where $G_{\mathcal{S}}(\mathcal{X})$ is the Gram matrix (see (4.3)), is biorthogonal to $\left\{\boldsymbol{g}_{k}\right\}_{k=1}^{n-1}$ and is known as the contravariant basis:

$$
\left\langle\boldsymbol{g}_{j}, \boldsymbol{g}^{k}\right\rangle=\delta_{j k}, \quad j, k=1, \ldots, n-1 .
$$

$\boldsymbol{\nu}(\mathcal{X})$ is the outer unit normal vector field to $\mathcal{S}$, which has the most important role in the calculus of tangent differential operators we are going to apply.

The next definition of a hypersurface, an implicit one, is equivalent with the definition given in (4.1)(4.2).

Definition 4.1. Let $k \geq 1$ and $\omega \subset \mathbb{R}^{n}$ be a compact domain. An implicit $C^{k}$-smooth (an implicit Lipschitz) hypersurface in $\mathbb{R}^{n}$ is defined as the set

$$
\mathcal{S}=\left\{\mathcal{X} \in \omega: \Psi_{\mathcal{S}}(\mathcal{X})=0\right\},
$$

where $\Psi_{\mathcal{S}}: \omega \rightarrow \mathbb{R}$ is a $C^{k}$-mapping (or Lipschitz mapping) which is regular: $\nabla \Psi(\mathcal{X}) \neq 0$.

It is well known that using implicit surface functions gradient from (4.7) we can write an alternative definition of the unit normal vector field on the surface (see (4.5)):

$$
\boldsymbol{\nu}(\mathcal{X}):=\lim _{x \rightarrow \mathcal{X}} \frac{\left(\nabla \Psi_{\mathcal{S}}\right)(x)}{\left|\left(\nabla \Psi_{\mathcal{S}}\right)(x)\right|}, \quad \mathcal{X} \in \mathcal{S} .
$$

Let $\mathcal{N}(x)=\left(\mathcal{N}_{1}(x), \mathcal{N}_{2}(x), \mathcal{N}_{3}(x)\right)^{\top}$ be an extension in the neighbourhood $U_{\mathcal{C}}$ of the surface $\mathcal{C}$ of the normal vector field $\boldsymbol{\nu}(\mathcal{X})=\left(\nu_{1}(\mathcal{X}), \nu_{2}(\mathcal{X}), \nu_{3}(\mathcal{X})\right)^{\top}$ on $\mathcal{C}$. 
Definition 4.2. A vector field $\mathcal{N} \in C^{1}\left(\Omega_{\mathcal{S}}\right)$ in a neighborhood $\Omega_{\mathcal{S}}$ of $\mathcal{S}$ will be referred to as a proper extension if $\left.\mathcal{N}\right|_{\mathcal{S}}=\boldsymbol{\nu}$, it is unitary $(|\mathcal{N}|=1)$ in $\Omega_{\mathcal{S}}$ and if $\mathcal{N}$ satisfies the condition

$$
\partial_{j} \mathcal{N}_{k}(x)=\partial_{k} \mathcal{N}_{j}(x) \text { for all } x \in \Omega_{\mathcal{S}}, \quad j, k=1, \ldots, n .
$$

In [11] is proved that there exists a unique proper extension of the normal vector field $\boldsymbol{\nu}(\mathcal{X})=$ $\left(\nu_{1}(\mathcal{X}), \nu_{2}(\mathcal{X}), \nu_{3}(\mathcal{X})\right)^{\top}, \mathcal{X} \in \mathcal{C}$, from the surface $\mathcal{C}$ in some neighbourhood of the surface.

Let

$$
\boldsymbol{P}(\nabla) u=\sum_{j=1}^{n} a_{j} \partial_{j} u+b u, \quad a_{j}, b \in C^{1}\left(\mathbb{R}^{m \times m}\right),
$$

be a first-order differential operator with real-valued (variable) matrix coefficients, acting on vectorvalued functions $\left(u=\left(u_{\beta}\right)_{\beta=1}^{n}\right)$ in $\mathbb{R}^{n}$, and its principal symbol is given by the matrix-valued function

$$
\sigma(\boldsymbol{P} ; \xi):=\sum_{j=1}^{n} a_{j} \xi_{j}, \quad \xi=\left\{\xi_{j}\right\}_{j=1}^{n} \in \mathbb{R}^{n} .
$$

We say that $\boldsymbol{P}$ is a tangent operator to $\mathcal{S}$ if the symbol vanishes on the normal vector field of the surface:

$$
\sigma(\boldsymbol{P} ; \boldsymbol{\nu})=0 \quad \text { on } \quad \mathcal{S} .
$$

The most important tangent differential operators to the hypersurface for us are Günter derivatives

$$
\mathcal{D}_{j}:=\partial_{j}-\nu_{j} \partial_{\nu}=\partial_{j}-\nu_{j} \sum_{k=1}^{n} \nu_{k} \partial_{k}, \quad j=1, \ldots, n .
$$

Günters derivatives are derivation along the tangent vectors to the surface

$$
\begin{gathered}
\mathcal{D}_{j}:=\partial_{\boldsymbol{d}^{j}}=\boldsymbol{d}^{j} \cdot \nabla, \\
\boldsymbol{d}^{j}:=\pi_{\mathcal{S}} \boldsymbol{e}^{j}:=\boldsymbol{e}^{j}-\nu_{j} \boldsymbol{\nu}=\sum_{k=1}^{n}\left(\delta_{j k}-\nu_{j} \nu_{k}\right) \boldsymbol{e}^{k}, \quad j=1, \ldots, n,
\end{gathered}
$$

where $\pi_{\mathcal{S}}$ is the projection on the tangent space to the surface and $\boldsymbol{e}^{j}$ is the canonical basis in the Euclidean space $\mathbb{R}^{n}$ :

$$
\boldsymbol{e}^{1}:=(1,0, \ldots, 0)^{\top}, \ldots, \boldsymbol{e}^{n}:=(0, \ldots, 0,1)^{\top}
$$

Therefore, $\mathcal{D}_{j}$ can be applied to functions which are defined only on the surface $\mathcal{S}$.

Note that in a strongly tangent operator the coordinate derivatives $\partial_{j}$ can be replaced by the Günter's derivatives $\mathcal{D}_{j}$ :

$$
\boldsymbol{P}(\nabla) u=\sum_{j=1}^{n} a_{j} \partial_{j} u+b u=\sum_{j=1}^{n} a_{j} \mathcal{D}_{j} u+b u=\boldsymbol{P}(\mathcal{D}) u, \quad a_{j}, b \in C^{1}\left(\mathbb{R}^{m \times m}\right) .
$$

The generating vector field $\left\{\boldsymbol{d}^{j}\right\}_{j=1}^{n}$ is not a frame since it is linearly dependent, as well as Günter's derivatives:

$$
\sum_{j=1}^{n} \nu_{j}(\mathcal{X}) \boldsymbol{d}^{j}(\mathcal{X}) \equiv 0, \quad \sum_{j=1}^{n} \nu_{j}(\mathcal{X}) \mathcal{D}_{j} \equiv 0 .
$$


Consider the following differential 1-form $\omega_{f}$

$$
\omega_{f}(V):=\mathcal{L}_{V} f=\sum_{k=1}^{n-1} V^{k} \partial_{k} f \quad \text { for } \quad f \in C^{1}(\mathcal{S}), \quad V=\sum_{k=1}^{n-1} V^{k} \boldsymbol{g}_{k} \in \mathcal{T S} .
$$

The form is correctly defined because the differential operator $\mathcal{L}_{V}$ is tangential and can be applied to a function $f$ defined on the surface $\mathcal{S}$ only.

Then, for a given $f$, there exists a vector field $\nabla_{\mathcal{S}} f \in \mathcal{T S}$ such that

$$
\omega_{f}(V):=\left(\nabla_{\mathcal{S}} f, V\right) \text { for all } V \in \mathcal{V}(X),
$$

which is, in classical differential geometry, the definition of surface Gradient of a function $f \in \mathbb{C}^{1}(\mathcal{S})$ and maps

$$
\nabla_{\mathcal{S}}: \mathbb{C}^{\infty}(\mathcal{S}) \rightarrow \mathcal{T S}
$$

The surface divergence

$$
\operatorname{div}_{\mathcal{S}}: \mathcal{V}(\mathcal{S}) \rightarrow \mathbb{C}^{\infty}(\mathcal{S})
$$

of a smooth tangent vector field is, by the definition, the dual operator with the opposite sign

$$
\left(\operatorname{div}_{\mathcal{S}} V, f\right):=-\left(V, \nabla_{\mathcal{S}} f\right), \quad \forall V \in \mathcal{T S}, \quad \forall f \in \mathbb{C}^{1}(\mathcal{S})
$$

These operators recorded in intrinsic parameters of the surface $\mathcal{S}$ (tangent vector fields, Metric tensor etc.) have rather complicated form. In [8] was proved the following.

Theorem 4.3. For any function $\varphi \in C^{1}(\mathcal{S})$ we have

$$
\nabla_{\mathcal{S}} \varphi=\left\{\mathcal{D}_{1} \varphi, \mathcal{D}_{2} \varphi, \ldots, \mathcal{D}_{n} \varphi\right\}^{\top}
$$

Also, for a 1-smooth tangent vector field $\boldsymbol{V}=\sum_{j=1}^{n} V^{j} e_{j} \in \omega(\mathcal{S})$,

$$
\operatorname{div}_{\mathcal{S}} \boldsymbol{V}=-\nabla_{\mathcal{S}}^{*} \boldsymbol{V}:=\sum_{j=1}^{n} \mathcal{D}_{j} V^{j}, \quad \operatorname{div}_{\mathcal{S}}^{*}=-\nabla_{\mathcal{S}}
$$

The Laplace-Beltrami operator $\boldsymbol{\Delta}_{\mathcal{S}}$ on $\mathcal{S}$ takes the form

$$
\boldsymbol{\Delta}_{\mathcal{S}} \psi=\operatorname{div}_{\mathcal{S}} \nabla_{\mathcal{S}} \psi=-\nabla_{\mathcal{S}}^{*}\left(\nabla_{\mathcal{S}} \psi\right)=\sum_{j=1}^{n} \mathcal{D}_{j}^{2} \psi \quad \forall \psi \in C^{2}(\mathcal{S}) .
$$

Corollary 4.4 (cf. [8]). Let $\mathcal{S}$ be a smooth closed hypersurface. The homogeneous equation

$$
\Delta_{\mathcal{S}} \psi=0
$$

has only a constant solution in the space $\mathbb{W}^{1}(\mathcal{S})$.

Proposition 4.5 (cf. [9]). For the operator $\operatorname{div}_{\mathcal{C}} \mathcal{A} \nabla_{\mathcal{C}}$ on the open hypersurface $\mathcal{C}$ with the boundary $\partial \mathcal{C}:=\Gamma$ the following I and II Green formulae are valid

$$
\begin{aligned}
\left(\operatorname{div}_{\mathcal{C}} \mathcal{A} \nabla_{\mathcal{C}} \varphi, \psi\right)_{\mathcal{C}} & =\left(\left\langle\boldsymbol{\nu}_{\Gamma}(s), \mathcal{A}\left(\nabla_{\mathcal{C}} u\right)^{+}(s)\right\rangle, \psi^{+}\right)_{\Gamma}-\left(\mathcal{A} \nabla_{\mathcal{C}} \varphi, \nabla_{\mathcal{C}} \psi\right)_{\mathcal{C}} \\
\left(\operatorname{div}_{\mathcal{C}} \mathcal{A} \nabla_{\mathcal{C}} \varphi, \psi\right)_{\mathcal{C}} & -\left(\varphi, \operatorname{div}_{\mathcal{C}} \mathcal{A} \nabla_{\mathcal{C}} \psi\right)_{\mathcal{C}} \\
& \left.=\left\langle\boldsymbol{\nu}_{\Gamma}(s), \mathcal{A}\left(\nabla_{\mathcal{C}} u\right)^{+}(s)\right\rangle, \psi^{+}\right)_{\Gamma}-\left(\varphi^{+},\left\langle\boldsymbol{\nu}_{\Gamma}(s), \mathcal{A}\left(\nabla_{\mathcal{C}} u\right)^{+}(s)\right\rangle\right)_{\Gamma},
\end{aligned}
$$


where $(\varphi, \psi)_{\mathcal{C}}$ denotes the scalar product of functions in $\mathbb{L}_{2}(\mathcal{C}),\langle\xi, \eta\rangle$ denotes the scalar product of vectors in $\mathbb{R}^{n}$ in and $\left\langle\boldsymbol{\nu}_{\Gamma}(s), \mathcal{A}\left(\nabla_{\mathcal{C}} u\right)^{+}(s)\right\rangle$ is the Neumann trace operator.

In particular, for the Laplace-Beltrami operator the following I and II Green formulae are valid

$$
\begin{gathered}
\left(\Delta_{\mathcal{C}} \varphi, \psi\right)_{\mathcal{C}}=\left(\left(\partial_{\nu_{\Gamma}} \varphi\right)^{+}, \psi^{+}\right)_{\Gamma}-\left(\nabla_{\mathcal{C}} \varphi, \nabla_{\mathcal{C}} \psi\right)_{\mathcal{C}}, \\
\left(\Delta_{\mathcal{C}} \varphi, \psi\right)_{\mathcal{C}}-\left(\varphi, \Delta_{\mathcal{C}} \psi\right)_{\mathcal{C}}=\left(\left(\partial_{\nu_{\Gamma}} \varphi\right)^{+}, \psi^{+}\right)_{\Gamma}-\left(\varphi^{+},\left(\partial_{\nu_{\Gamma}} \varphi\right)^{+}\right)_{\Gamma},
\end{gathered}
$$

where $\left(\partial_{\nu_{\Gamma}} \varphi\right)^{+}:=\left\langle\nu_{\Gamma},\left(\nabla_{\mathcal{C}} \varphi\right)^{+}\right\rangle$is the Neumann trace operator.

\section{Solvability of BVPs for the anisotropic Laplace-Beltrami equation on a hypersurface in the classical setting}

In the present section we investigate solvability of the Basic BVPs for the "anisotropic" LaplaceBeltrami equation. We use the notation introduced in (1.1)-(1.3) and in Section 3: $\mathcal{A}$ is a positive definite $n \times n$ matrix function (see (1.2)), $\operatorname{div}_{\mathcal{C}}$ is the surface divergence and $\boldsymbol{\nabla}_{\mathcal{C}}$ is the surface gradient (see (4.23) and (4.24)).

We study solvability of the Mixed BVP

$$
\begin{cases}\operatorname{div}_{\mathcal{C}} \mathcal{A} \nabla_{\mathcal{C}} u(\mathcal{X})=f(\mathcal{X}), & \mathcal{X} \in \mathcal{C}, \\ u^{+}(s)=g(s), & \text { on } \Gamma_{D}, \\ \left\langle\boldsymbol{\nu}_{\Gamma}(s), \mathcal{A}\left(\nabla_{\mathcal{C}} u\right)^{+}(s)\right\rangle=h(s), & \text { on } \Gamma_{N},\end{cases}
$$

of the Dirichlet BVP

$$
\begin{cases}\operatorname{div}_{\mathcal{C}} \mathcal{A} \nabla_{\mathcal{C}} u(\mathcal{X})=f(\mathcal{X}), & \mathcal{X} \in \mathcal{C}, \\ u^{+}(s)=g(s), & \text { on } \Gamma\end{cases}
$$

and of the Neumann BVP

$$
\begin{cases}\operatorname{div}_{\mathcal{C}} \mathcal{A} \nabla_{\mathcal{C}} u(\mathcal{X})=f(\mathcal{X}), & \mathcal{X} \in \mathcal{C}, \\ \left\langle\boldsymbol{\nu}_{\Gamma}(s), \mathcal{A}\left(\nabla_{\mathcal{C}} u\right)^{+}(s)\right\rangle=h(s), & \text { on } \quad \Gamma .\end{cases}
$$

Here $\boldsymbol{\nu}_{\Gamma}(\mathcal{X})$ denotes the outer normal vector field to the boundary $\Gamma$, which is tangent to $\mathcal{S}$ and $\left\langle\boldsymbol{\nu}_{\Gamma}(s), \mathcal{A}\left(\nabla_{\mathcal{C}} u\right)\right.$ denotes the "Neumann trace operator, which appears in the Green formula (4.27)). For $\mathcal{A}(\mathcal{X}) \equiv 1$, the operator $\operatorname{div}_{\mathcal{C}} \mathcal{A} \nabla_{\mathcal{C}}$ becomes the Laplace-Beltrami operator $\operatorname{div}_{\mathcal{C}} \nabla_{\mathcal{C}}=\Delta_{\mathcal{C}}$ and the Neumann trace operator becomes the trace of normal derivative $\left(\partial_{\boldsymbol{\nu}_{\Gamma}} u\right)^{+}=\left\langle\boldsymbol{\nu}_{\Gamma},\left(\nabla_{\mathcal{C}} u\right)^{+}\right\rangle$.

The BVPs (5.1)-(5.3) are investigated in the following classical weak settings in the spaces of $n$-vector functions

$$
u \in \mathbb{H}^{1}(\mathcal{C}), \quad f \in \widetilde{\mathbb{H}}_{0}^{-1}(\mathcal{C}), \quad g \in \mathbb{H}^{\frac{1}{2}}\left(\Gamma_{D}\right), \quad h \in \mathbb{H}^{-\frac{1}{2}}\left(\Gamma_{N}\right), \quad \Gamma=\Gamma_{D} \cup \Gamma_{N}
$$

for the mixed BVP (also cf. (1.2)), and in the weak settings

$$
f \in \widetilde{\mathbb{H}}^{-1}(\mathcal{C}), \quad g \in \mathbb{H}^{1 / 2}(\Gamma), \quad h \in \mathbb{H}^{-1 / 2}(\Gamma)
$$

for the Dirichlet and Neumann BVPs.

The main objective of the present section is to prove the following 
Theorem 5.1. Let $\mathcal{C} \subset \mathbb{R}^{3}$ be a hypersurface with the Lipschitz boundary $\Gamma:=\partial \mathcal{C}$.

The Mixed BVP (5.1) in the classical setting (5.4) has a unique solution.

The Dirichlet BVP (5.2) in the classical setting (5.5) has a unique solution.

For the solvability of the Neumann problem (5.3) in the classical setting (5.5) the following necessary and sufficient compatibility condition has to hold:

$$
(f, 1)_{\mathcal{C}}=(h, 1)_{\Gamma}
$$

Note that if $f$ and $h$ are regular integrable functions, the compatibility condition (5.6) acquires the form

$$
\int_{\mathcal{C}} f(y) d \sigma-\oint_{\Gamma} h(s) d s=0 .
$$

The formulated theorem will be proved at the end of the present section. Prior to this, we will expose auxiliary material for the proof.

Theorem 5.2. Let $\mathcal{S}$ be $\ell$-smooth $\ell=1,2, \ldots, 1<p<\infty,|s| \leqslant \ell$ and $\mathcal{A}(\mathcal{X})$ be a positive definite $3 \times 3$ matrix function. Let $\mathbb{X}_{p}^{s}(\mathcal{S})$ be the same vector-space as above.

Let the matrix-function $\mathcal{H} \in\left[C^{\ell-1}\left(\mathbb{R}^{n}\right)\right]^{3 \times 3}$ have one of the following properties:

i. $\mathcal{H}$ has a non-negative definite real part $(\operatorname{Re} \mathcal{H} \varphi, \varphi)_{\mathcal{C}} \geqslant 0$ and mes $\operatorname{supp} \operatorname{Re} \mathcal{H} \neq 0$;

ii. mes $\operatorname{supp} \operatorname{Im} \mathcal{H} \neq 0$ and the complex part $\operatorname{Im} \mathcal{H}$ is either positive definite or negative definite:

$$
(\varepsilon \operatorname{Im} \mathcal{H} \varphi, \varphi)_{\mathcal{C}} \geqslant C>0 \text { for all }\left\|\varphi \mid L_{2}(\mathcal{C})\right\|=1,
$$

where $\varepsilon=1$ or $\varepsilon=-1$.

Then the perturbed operator

$$
\operatorname{div}_{\mathcal{S}} \mathcal{A} \nabla_{\mathcal{S}}-\mathcal{H} I: \mathbb{X}_{p}^{s+1}(\mathcal{S}) \rightarrow \mathbb{X}_{p}^{s-1}(\mathcal{S})
$$

is invertible.

Moreover, the operator $\operatorname{div}_{\mathcal{S}} \mathcal{A} \nabla_{\mathcal{S}}$ is also invertible in the space setting with detached constants (see (2.3))

$$
\operatorname{div}_{\mathcal{S}} \mathcal{A} \nabla_{\mathcal{S}}: \mathbb{X}_{p, \#}^{s+1}(\mathcal{S}) \rightarrow \mathbb{X}_{p, \#}^{s-1}(\mathcal{S})
$$

and, therefore, $\operatorname{div}_{\mathcal{S}} \mathcal{A} \nabla_{\mathcal{S}}$ has the fundamental solution in setting (5.10).

The invertibility is also interpreted as the existence of the fundamental solution to the operators $\operatorname{div}_{\mathcal{S}} \mathcal{A} \nabla_{\mathcal{S}}-\mathcal{H} I$ and $\operatorname{div}_{\mathcal{S}} \mathcal{A} \nabla_{\mathcal{S}}$ in the corresponding spaces.

In particular, the perturbed Laplace-Beltrami operator $\Delta_{\mathcal{S}}-\mathcal{H} I$ (the particular case $\mathcal{A}(\mathcal{X}) \equiv 1$ ) is invertible in setting (5.9) (has the fundamental solution), while the Laplace-Beltrami operator $\Delta_{\mathcal{S}}$ is invertible in setting (5.10) (has the fundamental solution). 
Proof: First of all note that the operators in (5.8) and (5.9) are bounded. For the operator in (5.8) this is trivial, while for the operator in (5.9) we need to check that the image of the operator belongs to the subspace $\mathbb{X}_{p}^{s-1}(\mathcal{S})$, i.e., is orthogonal to the identity 1 (see (2.3)). Indeed, by applying formula (4.24) we get

$$
\left(\operatorname{div}_{\mathcal{S}} \mathcal{A} \nabla_{\mathcal{S}} \varphi, 1\right)_{\mathcal{S}}=\left(\mathcal{A} \nabla_{\mathcal{S}} \varphi, \nabla_{\mathcal{S}} 1\right)_{\mathcal{S}}=0
$$

This operator in the setting

$$
\operatorname{div}_{\mathcal{S}} \mathcal{A} \nabla_{\mathcal{S}}: \mathbb{X}_{2, \#}^{1}(\mathcal{S}) \rightarrow \mathbb{X}_{2, \#}^{-1}(\mathcal{S})
$$

is coercive:

$$
-\left(\operatorname{div}_{\mathcal{S}} \mathcal{A} \nabla_{\mathcal{S}} \varphi, \varphi\right)_{\mathcal{S}}=\left(\mathcal{A} \nabla_{\mathcal{S}} \varphi, \nabla_{\mathcal{S}} \varphi\right)_{\mathcal{S}} \geqslant C\left\|\varphi \mid \mathbb{X}_{2 \#}^{1}(\mathcal{S})\right\|^{2}
$$

Then, due to Corollary 3.4, this operator is invertible in the setting (5.11).

Moreover, this operator $\operatorname{div}_{\mathcal{S}} \mathcal{A} \nabla_{\mathcal{S}}$ is elliptic and even has negative definite symbol $\xi^{\top} \mathcal{A} \xi, \xi \in \mathbb{R}^{m}$ (ellipticity follows from the invertibility in setting (5.12), as well). As an elliptic operator on the closed hypersurface the operator in (5.10) is Fredholm for all $s \in \mathbb{R}$ and $1<p<\infty$ (it has a parametrix if $\mathcal{S}$ is infinitely smooth, see $[18,25,28]$ for a similar arguments). Since all operators in the homotopy

$$
\mathbf{B}_{\lambda}=(1-\lambda) \operatorname{div}_{\mathcal{S}} \mathcal{A} \nabla_{\mathcal{S}}-\lambda \Lambda^{2}(\mathcal{X}, D), \quad 0 \leqslant \lambda \leqslant 1
$$

where

$$
\Lambda_{2}(\mathcal{X}, D): \mathbb{X}_{p, \#}^{s+1}(\mathcal{S}) \rightarrow \mathbb{X}_{p, \#}^{s-1}(\mathcal{S})
$$

is the Bessel potential operator with positive definite symbol and arrange the isometrical isomorphism of the spaces (see Corollary (3.4)), have positive definite symbol, they are Fredholm operators in the setting

$$
\mathbf{B}_{\lambda}: \mathbb{X}_{p, \#}^{s+1}(\mathcal{S}) \rightarrow \mathbb{X}_{p, \#}^{s-1}(\mathcal{S})
$$

for all $0 \leqslant \lambda \leqslant 1,1<p<\infty,|s| \leqslant \ell$. Then

$$
\text { Ind } \operatorname{div}_{\mathcal{S}} \mathcal{A} \nabla_{\mathcal{S}}=\text { Ind } \mathbf{B}_{0}=\text { Ind } \mathbf{B}_{\lambda}=\text { Ind } \mathbf{B}_{1}=\text { Ind } \lambda^{2}(\mathcal{X}, D)=0
$$

and Theorem 3.6 can be applied, which states that the operator in (5.10) is invertible for all $1<p<\infty$, $|s| \leqslant \ell$.

Since the operator in (5.9) is the perturbation by lower order operator $\mathcal{H} I$ (i.e., by a compact operator) of the invertible operator in (5.9), the operator in (5.9) is Fredholm and has trivial index $\operatorname{Ind}\left[\operatorname{div}_{\mathcal{S}} \mathcal{A} \nabla_{\mathcal{S}}-\right.$ $\mathcal{H}]=0$. Then to prove that the operator in (5.9) is invertible we just need to check that it has trivial kernel, i.e., if $\left(\operatorname{div}_{\mathcal{S}} \mathcal{A} \nabla_{\mathcal{S}}-\mathcal{H}\right) \varphi=0$, then $\varphi=0$. Due to equality (4.24),

$$
\begin{aligned}
\left(-\left(\operatorname{div}_{\mathcal{S}} \mathcal{A} \nabla_{\mathcal{S}}-\mathcal{H}\right) \varphi, \varphi\right)_{\mathcal{S}} & \\
& =\left(\mathcal{A} \nabla_{\mathcal{S}} \varphi, \nabla_{\mathcal{S}} \varphi\right)_{\mathcal{S}}+(\operatorname{Re} \mathcal{H} \varphi, \varphi)_{\mathcal{S}}+i(\operatorname{Im} \mathcal{H} \varphi, \varphi)_{\mathcal{S}}, \quad \forall \varphi \in \mathbb{W}_{2}^{1}(\mathcal{S})
\end{aligned}
$$

If $\varphi$ is a solution to the homogeneous equation $\left(\operatorname{div}_{\mathcal{S}} \mathcal{A} \nabla_{\mathcal{S}}-\mathcal{H}\right) \varphi=0$, equality (5.10) takes the form

$$
\left(\mathcal{A} \nabla_{\mathcal{S}} \varphi, \nabla_{\mathcal{S}} \varphi\right)_{\mathcal{S}}+(\operatorname{Re} \mathcal{H} \varphi, \varphi)_{\mathcal{S}}=0, \quad(\operatorname{Im} \mathcal{H} \varphi, \varphi)_{\mathcal{S}}=0
$$


Now let $\operatorname{Re} \mathcal{H}(\mathcal{X}) \geqslant 0$ for all $\mathcal{X} \in \mathcal{S}$ and mes supp $\operatorname{Re} \mathcal{H} \neq 0$ (case (i)). Then from the first equality in (5.13) it follows

$$
C\left\|\nabla_{\mathcal{S}} \varphi\right\| \leqslant\left(\mathcal{A} \nabla_{\mathcal{S}} \varphi, \nabla_{\mathcal{S}} \varphi\right)_{\mathcal{S}}=0, \quad(\operatorname{Re} \mathcal{H} \varphi, \varphi)_{\mathcal{S}}=0
$$

(the inequality is due to the positive definiteness of $\mathcal{A}$ ). From the first inequality we get $\nabla_{\mathcal{S}} \varphi \equiv 0$ and, consequently, $\varphi=C=$ const (this is easy to ascertain by analysing the definition of Günter's derivatives; see, e.g., [9]). By inserting this in the second equality in (5.13) we get

$$
0=(\operatorname{Re} \mathcal{H} \varphi, \varphi)_{\mathcal{S}}=C \int_{\mathcal{S}} \mathcal{H}(\mathcal{X}) d \sigma
$$

and the conclusion $\varphi(\mathcal{X})=C=0$ is immediate, because mes supp $\operatorname{Re} \mathcal{H} \neq 0$.

In the case (ii), from the second equality in (5.14) we have

$$
C\|\varphi\| \leqslant \varepsilon\left(\operatorname{Im} \mathcal{H} \varphi, \nabla_{\mathcal{S}} \varphi\right)_{\mathcal{S}}=0
$$

(the inequality is due to the positive definiteness of $\varepsilon \operatorname{Im} \mathcal{H}$ ) and, again, $\varphi=0$.

Note that a function $\varphi \in \mathbb{W}_{p}^{s}(\mathcal{C})$ (and $\varphi \in \mathbb{H}_{p}^{s}(\mathcal{C})$ ) has the trace $\varphi^{+} \in \mathbb{W}_{p}^{s-\frac{1}{p}}(\Gamma)$ on the boundary, provided $1<p<\infty$ and $s>\frac{1}{p}$ (see [30] for details). Therefore, if we look for a solution to Dirichlet $\operatorname{BVP}(5.2)$ in the space $\mathbb{W}^{1}(\mathcal{C})$, the trace $u^{+}$on $\Gamma_{D}$ exists and belongs to the space $\mathbb{H}^{1 / 2}\left(\Gamma_{D}\right)$.

Concerning the existence of the Neumann trace $\left\langle\boldsymbol{\nu}_{\Gamma}, \mathcal{A} \nabla_{\mathcal{C}} u\right\rangle^{+}$in (5.1) and (5.3) for a solution $u \in$ $\mathbb{W}^{1}(\mathcal{C})$, it is not guaranteed by the general trace theorem. But in this case, the first Green formula (4.27) ensures the existence of the Neumann trace. Indeed, by setting $\varphi=u$ and inserting the data $\left(\operatorname{div}_{\mathcal{C}} A \nabla_{\mathcal{C}}\right) u(\mathcal{X})=f(\mathcal{X})$ from (5.1) into the first Green formula (4.27) we obtain

$$
\left(\left\langle\boldsymbol{\nu}_{\Gamma},\left(\mathcal{A} \nabla_{\mathcal{C}} u\right)^{+}\right\rangle, \psi^{+}\right)_{\Gamma}-\left(\mathcal{A} \nabla_{\mathcal{C}} u, \nabla_{\mathcal{C}} \psi\right)_{\mathcal{C}}=\left(\operatorname{div}_{\mathcal{C}}\left(\mathcal{A} \nabla_{\mathcal{C}} u\right), \psi\right)_{\mathcal{C}}=(f, \psi)_{\mathcal{C}}
$$

and, finally, we get

$$
\left(\left\langle\boldsymbol{\nu}_{\Gamma},\left(\mathcal{A} \nabla_{\mathcal{C}} u\right)^{+}\right\rangle, \psi^{+}\right)_{\Gamma}=\left(\mathcal{A} \nabla_{\mathcal{C}} u, \nabla_{\mathcal{C}} \psi\right)_{\mathcal{C}}+(f, \psi)_{\mathcal{C}}
$$

for arbitrary $\psi \in \mathbb{W}^{1}(\mathcal{C})$. Since $\psi^{+} \in \mathbb{H}^{1 / 2}(\Gamma)$, the scalar product $\left(\mathcal{A} \nabla_{\mathcal{C}} u, \nabla_{\mathcal{C}} \psi\right)_{\mathcal{C}}$ in the right-hand side of equality (5.15) is correctly defined and defines correct duality in the left-hand side of the equality. Since $\psi^{+} \in \mathbb{H}^{1 / 2}(\Gamma)$ is arbitrary, by the duality argument this gives that $\left\langle\boldsymbol{\nu}_{\Gamma},\left(\mathcal{A} \nabla_{\mathcal{C}} u\right)^{+}\right\rangle$should be in the dual space, i.e., in $\mathbb{H}^{-1 / 2}(\Gamma)$.

To prove the above formulated Theorem 5.1, we need more properties of trace operator (called retraction) and their inverses, called co-retractions (see [30, $§ 2.7]$ ).

To keep the exposition simpler we recall a very particular case of Lemma 4.8 from [7], which we apply in the present investigation.

A differential operator

$$
\boldsymbol{B}(\mathcal{X}, \mathcal{D})=\sum_{|\alpha| \leqslant m} a_{\alpha}(\mathcal{X}) \mathcal{D}^{\alpha}, \quad \mathcal{X} \in \mathcal{C}
$$


on a hypersurface $\mathcal{C} \subset \mathbb{R}^{3}$ with the Lipschitz boundary $\Gamma=\partial \mathcal{C}$ is called normal if its symbol (the characteristic polynomial)

$$
\mathcal{B}(\mathcal{X}, \xi)=\sum_{|\alpha| \leqslant m} a_{\alpha}(\mathcal{X})(-i \xi)^{\alpha}, \quad \mathcal{X} \in \mathcal{C}, \quad \xi \in \mathbb{R}^{3}
$$

does not vanish on normal vactor field on the boundary $\inf _{s \in \Gamma}|\operatorname{det} \mathcal{B}(s, \boldsymbol{\nu}(s))|>0$.

Proposition 5.3 (see Lemma 4.8 in [7]). Let $s \notin \mathbb{N}, 1<p<\infty$, and $\mathcal{C}$ be a hypersurface with the Lipschitz boundary $\Gamma=\partial \mathcal{C}$. Further, let $s>0, \boldsymbol{B}(D)$ be a normal differential operator of the first order defined in the vicinity of the boundary $\Gamma$ and $\boldsymbol{A}(D)$ be a normal differential operator of the second order defined on the surface $\mathcal{C}$. Then there exists a continuous linear operator

$$
\mathcal{R}: \mathbb{W}_{p}^{s}(\Gamma) \otimes \mathbb{W}_{p}^{s-1}(\Gamma) \longrightarrow \mathbb{H}_{p}^{s+\frac{1}{p}}(\mathcal{C})
$$

such that

$$
(\mathcal{R} \Phi)^{+}=\varphi_{0}, \quad(\boldsymbol{B}(D) \mathcal{R} \Phi)^{+}=\varphi_{1}, \quad \boldsymbol{A}(D) \mathcal{B} \Phi \in \widetilde{\mathbb{H}}_{p}^{s-2+\frac{1}{p}}(\mathcal{C})
$$

for arbitrary pair of functions $\Phi=\left(\varphi_{0}, \varphi_{1}\right)^{\top}$, where $\varphi_{0} \in \mathbb{W}_{p}^{s}(\Gamma)$ and $\varphi_{1} \in \mathbb{W}_{p}^{s-1}(\Gamma)$.

Proof of Theorem 5.1: We commence by the reduction of the BVP (5.1) to an equivalent one with the homogeneous Dirichlet condition. For this, we extend the boundary data $g \in \mathbb{W}^{1 / 2}\left(\Gamma_{D}\right)$ up to some function $\widetilde{g} \in \mathbb{W}^{1 / 2}(\Gamma)$ on the entire boundary $\Gamma$ and apply Proposition 5.3: there exists a function $G \in \mathbb{W}^{1}(\mathcal{C})$ such that $G^{+}(\mathcal{X})=g(\mathcal{X})$ for $\mathcal{X} \in \Gamma_{D}$ (actually $G^{+}=\widetilde{g}$ almost everywhere on the boundary) and $\operatorname{div}_{\mathcal{C}}\left(\mathcal{A} \nabla_{\mathcal{C}} G\right) \in \widetilde{\mathbb{W}}^{-1}(\overline{\mathcal{C}})$.

For a new unknown function $v:=u-G$ we have the following equivalent reformulation of the BVP (5.1):

$$
\begin{cases}\operatorname{div}_{\mathcal{C}}\left(\mathcal{A} \nabla_{\mathcal{C}} v\right)(\mathcal{X})=f_{0}(\mathcal{X}), & \mathcal{X} \in \mathcal{C}, \\ v^{+}(s)=0, & \text { on } \Gamma_{D}, \\ \left\langle\boldsymbol{\nu}_{\Gamma}(s),\left(\mathcal{A} \nabla_{\mathcal{C}} v\right)^{+}(s)\right\rangle=h_{0}(s), & \text { on } \Gamma_{N},\end{cases}
$$

where

$$
\begin{aligned}
f_{0}:=f+\operatorname{div}_{\mathcal{C}}\left(\mathcal{A} \nabla_{\mathcal{C}} G\right) & \in \widetilde{\mathbb{W}}^{-1}(\mathcal{C}), \quad h_{0}:=h+\left\langle\boldsymbol{\nu}_{\Gamma},\left(\mathcal{A} \nabla_{\mathcal{C}} G\right)^{+}\right\rangle \in \mathbb{W}^{-1 / 2}(\Gamma) \\
& v^{+} \in \widetilde{\mathbb{W}}^{1}\left(\Gamma_{D}, \mathcal{C}\right) \mathbb{W}^{1 / 2}\left(\Gamma_{N}\right)
\end{aligned}
$$

To justify the last inclusion $v^{+} \in \widetilde{\mathbb{W}}^{1 / 2}\left(\Gamma_{N}\right)$ note that, due to our construction, the trace of a solution vanishes on $\Gamma_{D}:\left.v^{+}\right|_{\Gamma_{D}}=0$.

By inserting the data from the reformulated boundary value problem (5.18) into the first Green identity (4.27), where $\varphi=\psi=v$, we get

$$
\begin{aligned}
\left(\mathcal{A} \nabla_{\mathcal{C}} v, \nabla_{\mathcal{C}} v\right)_{\mathcal{C}} & =\left(\left\langle\boldsymbol{\nu}_{\Gamma},\left(\mathcal{A} \nabla_{\mathcal{C}} v\right)^{+}\right\rangle, v^{+}\right)_{\Gamma_{D}}+\left(\left\langle\boldsymbol{\nu}_{\Gamma},\left(\mathcal{A} \nabla_{\mathcal{C}} v\right)^{+}\right\rangle, v^{+}\right)_{\Gamma_{N}} \\
& -\left(\operatorname{div}_{\mathcal{C}}\left(\mathcal{A} \nabla_{\mathcal{C}} v\right), v\right)_{\mathcal{C}}=\left(h_{0}, v^{+}\right)_{\Gamma_{N}}-\left(f_{0}, v\right)_{\mathcal{C}}, \quad v \in \widetilde{\mathbb{W}}^{1}\left(\Gamma_{D}, \mathcal{C}\right) .
\end{aligned}
$$

In the left-hand side of equality (5.20) we have a symmetric bilinear form, which is positive definite

$$
\left(\mathcal{A} \nabla_{\mathcal{C}} v, \nabla_{\mathcal{C}} v\right)_{\mathcal{C}} \geqslant C\left\|\nabla_{\mathcal{C}} v\right\|^{2}=\left\|v \mid \widetilde{\mathbb{W}}^{1}\left(\Gamma_{D}, \mathcal{C}\right)\right\|^{2} \quad \forall v \in \widetilde{\mathbb{W}}^{1}\left(\Gamma_{D}, \mathcal{C}\right)
$$


because $\mathcal{A}(s)$ is strictly positive definite matrix and we have applied the equivalent norm (2.7) in the space $\widetilde{\mathbb{W}}^{1}\left(\Gamma_{D}, \mathcal{C}\right)$.

$\left(h_{0}, v^{+}\right)_{\Gamma_{N}}$ and $\left(f_{0}, v\right)_{\mathcal{C}}$ in equality (5.20) are correctly defined continuous functionals, because $h_{0} \in$ $\mathbb{W}^{-1 / 2}(\Gamma), f_{0} \in \widetilde{\mathbb{W}}^{-1}(\mathcal{C})$, while their counterparts in the functional belong to the dual spaces $v^{+} \in$ $\widetilde{\mathbb{W}}^{1 / 2}\left(\Gamma_{D}\right)$ and $v \in \widetilde{\mathbb{W}}^{1}\left(\Gamma_{N}, \mathcal{C}\right) \subset \mathbb{W}^{1}(\mathcal{C})$.

Application of the Lax-Milgram Lemma 3.7 accomplishes the proof of the unique solvability of the mixed BVP (5.1) in setting (5.4).

Now we prove the unique solvability of the Dirichlet BVP (5.2) in setting (5.5). We commence, as above, with an equivalent reformulation: due to Proposition 5.3, we can pick up a function $G \in \mathbb{W}^{1}(\mathcal{C})$ such that $G^{+}=g$ and $\operatorname{div}_{\mathcal{C}}\left(\mathcal{A} \nabla_{\mathcal{C}} G\right) \in \widetilde{\mathbb{W}}^{-1}(\mathcal{C})$.

For a new unknown function $v:=u-G$ we have the following equivalent reformulation of the BVP (5.2):

$$
\begin{cases}\operatorname{div}_{\mathcal{C}}\left(A \nabla_{\mathcal{C}} v\right)(\mathcal{X})=f_{0}(\mathcal{X}), & \mathcal{X} \in \mathcal{C}, \\ v^{+}(s)=0, & \text { on } \Gamma\end{cases}
$$

where

$$
f_{0}:=f+\operatorname{div}_{\mathcal{C}}\left(\mathcal{A} \nabla_{\mathcal{C}} G\right) \in \widetilde{\mathbb{W}}^{-1}(\mathcal{C}), \quad v \in \widetilde{\mathbb{W}}^{1}(\mathcal{C}) .
$$

By inserting the data from the reformulated boundary value problem (5.21) into the first Green identity (4.27), where $\varphi=\psi=v$, we get

$$
\left(A \nabla_{\mathcal{C}} v, \nabla_{\mathcal{C}} v\right)_{\mathcal{C}}=\left(\left\langle\boldsymbol{\nu}_{\Gamma},\left(A \nabla_{\mathcal{C}} v\right)^{+}\right\rangle, v^{+}\right)_{\Gamma}-\left(\operatorname{div}_{\mathcal{C}}\left(A \nabla_{\mathcal{C}} v\right), v\right)_{\mathcal{C}}=-\left(f_{0}, v\right)_{\mathcal{C}}, \quad v \in \widetilde{\mathbb{W}}^{1}(\mathcal{C}) .
$$

What we get is similar to identity (5.20) which we derived in the foregoing case: the positive definite form in the left-hand side and a single correctly defined functional in the right-hand side. Again, applying the Lax-Milgram Lemma 3.7, the unique solvability of the Dirichlet BVP (5.2) can be proved in setting (5.5).

In conclusion, we prove the unique solvability of the Neumann BVP (5.3) in setting (5.5). Let us insert the data from the boundary value problem (5.3) into the first Green identity (4.27), where $\varphi=\psi=u$. We get

$$
\begin{aligned}
\left(\mathcal{A} \nabla_{\mathcal{C}} u, \nabla_{\mathcal{C}} u\right)_{\mathcal{C}} & =\left(\left\langle\boldsymbol{\nu}_{\Gamma}, \mathcal{A} \nabla_{\mathcal{C}} u\right\rangle^{+}, u^{+}\right)_{\Gamma}-\left(\left(\operatorname{div}_{\mathcal{C}} \mathcal{A} \nabla_{\mathcal{C}}\right) u, u\right)_{\mathcal{C}} \\
& =(h, u)_{\Gamma}-(f, u)_{\mathcal{C}}, \quad u \in \mathbb{W}_{2, \#}^{1}(\mathcal{C}) .
\end{aligned}
$$

We have to look for a solution in the subspace $\mathbb{W}_{2, \#}^{1}(\mathcal{C})$ (see (2.3)) because the constants are trivial solutions of the homogeneous BVP (5.1) with $f=h=0$. Since identity (5.22) has to be valid for constant $u(\mathcal{X})=1$ and the left-hand side vanishes for such solution, we get the necessary condition of solvability $(h, 1)_{\Gamma}-(f, 1)_{\mathcal{C}}=0$, which is the compatibility condition (5.6).

In the left-hand side of equality (5.22) we have a symmetric bilinear form, which is positive definite

$$
\left(\mathcal{A} \nabla_{\mathcal{C}} v, \nabla_{\mathcal{C}} v\right)_{\mathcal{C}} \geqslant C\left\|\nabla_{\mathcal{C}} v\right\|^{2}=\left\|v \mid \mathbb{W}_{2, \#}^{1}(\mathcal{C})\right\|^{2} \quad \forall v \in \mathbb{W}_{2, \#}^{1}(\mathcal{C})
$$

because $\mathcal{A}(s)$ is strictly positive definite matrix and we have applied equivalent norms (2.6) in the space $\mathbb{W}_{2, \#}^{1}(\mathcal{C})$. 
Further, both functionals in the right-hand side of (5.22) are bounded on the subspace $\mathbb{W}_{2, \#}^{1}(\mathcal{C})$. Application of the Lax-Milgram Lemma 3.7 accomplishes the proof of the unique solvability of the Neumann BVP (5.3) in setting (5.5), provided the compatibility condition (5.5) holds.

\section{References}

[1] G. R. Allan, Holomorphic vector-valued functions on a domain of holomorphy. J. London Math. Soc. 42, 1967, 509513.

[2] J. Bergh, J. Löfström, Interpolation spaces. An introduction, Grundlehren der mathematischen Wissenschaften. 223. Heidelberg, Springer-Verlag. 1976.

[3] V. D. Didenko, B. Silbermann, Approximation of Additive Convolution-Like Operators. Real C*-Algebra Approach, Birkhäuser Verlag Basel, Boston, Berlin 2008.

[4] R. Douglas, Convolutions. Academic Press, Inc., Boston, MA, 1993.

[5] R. Duduchava, Integral Equations with Fixed Singularities, Teubner, Leipzig, 1979.

[6] R. Duduchava, On multidimensional singular integral operators. I: The half-space case; II: The case of compact manifolds. J. Oper. Theory 11 (1984), 41-76; 199-214.

[7] R. Duduchava, The Green formula and layer potentials. Integral Equations Operator Theory 41 (2001), no. 2, 127178.

[8] R. Duduchava, D. Mitrea and M. Mitrea, Differential operators and boundary value problems on hypersurfaces. Math. Nachr. 279 (2006), no. 9-10, 996-1023.

[9] R. Duduchava, Partial differential equations on hypersurfaces. Mem. Differential Equations Math. Phys. 48 (2009), $19-74$.

[10] R. Duduchava, Mixed type boundary value problems for Laplace-Beltrami equation on a surface with the Lipschitz boundary. Georgian Math. J., DOI: https://doi.org/10.1515/gmj-2020-2074 | Published online: 11 Aug 2020.

[11] R. Duduchava, E. Shargorodsky and G. Tephnadze, Extension of the unit normal vector field from a hypersurface. Georgian Math. J. 22 (2015), no. 3, 355-359.

[12] R. Duduchava, F.-O. Speck, Pseudodifferential operators on compact manifolds with Lipschitz boundary. Mathematische Nachrichten 160, 1993, 149-191.

[13] R. Duduchava and M. Tsaava, Mixed boundary value problems for the Laplace-Beltrami equation. Complex Var. Elliptic Equ. 63 (2018), no. 10, 1468-1496.

[14] R. Duduchava and M. Tsaava, Mixed boundary value problems for the Helmholtz equation in a model 2D angular domain. Georgian Math. J. 27 (2020), no. 2, 211-231.

[15] R. DuDuchava, M. Tsaava and T. Tsutsunava, Mixed boundary value problem on hypersurfaces. Int. J. Differ. Equ. 2014, Art. ID 245350, 8 pp.

[16] I. Gohberg and N. Krupnik, One-Dimensional Linear Singular Integral Equations I, Oper. Theory, Adv. Appl. 53, Birkhäuser Verlag, Basel, 1992.

[17] L. Hörmander, Estimates for translation invariant operators on $L^{p}$-spaces. Acta Mathematica 104, 1960, 93-140.

[18] L. Hörmander, The Analysis of Linear Partial Differential Operators. III. Pseudo-Differential Operators. Grundlehren der Mathematischen Wissenschaften [Fundamental Principles of Mathematical Sciences], 274. Springer-Verlag, Berlin, 1983.

[19] L.V. Kantorovich, G.P. Akilov, Functional analysis. Transl. from the Russian by Howard L. Silcock. 2nd ed. (English) Oxford etc.: Pergamon Press, Oxford-Elmsford, N.Y. 1982. 
[20] B. Khvedelidze, Linear discontinuous boundary value problems of function theory, singular integral equations and some of their applications. (Russian) em Trudy Tbiliss. Mat. Inst. 23 (1956), 3-158.

[21] M. Krasnosel'skij, On a theorem of M. Riesz, Sov. Math. Dokl. 1, 1960, 229-231; translation from Dokl. Akad. Nauk SSSR 131, 1960, 246-248.

[22] P. D. Lax and A. N. Milgram, Parabolic equations. In: Contributions to the theory of partial differential equations, pp. 167-190. Annals of Mathematics Studies, no. 33. Princeton University Press, Princeton, N. J., 1954.

[23] H. Le Dret. Numerical Approximation of PDEs, Master 1 Lecture Notes 2011-2012 http://www.ann.jussieu.fr/ ledret/M1ApproxPDE.html

[24] R.T. Seeley, Singular integrals and boundary value problems American Journal of Mathematics 88, 1966, 781-809.

[25] M. Shubin, Pseudodifferential Operators and Spectral Theory. Translated from the 1978 Russian original by Stig I. Andersson. Second edition. Springer-Verlag, Berlin, 2001.

[26] I.B. Simonenko, A new general method of investigating linear operator equations of the type of singular integral equations, Sov. Math., Dokl. 5 (1964), 1323-1326. Translation from Dokl. Akad. Nauk SSSR 158 (1964), 790-793.

[27] P. Solin, Partial differential equations and the finite element method, John Wiley \& Sons, Inc., New Jersey, 2006.

[28] M. E. Taylor, Partial Differential Equations. I. Basic Theory. Applied Mathematical Sciences, 115. Springer-Verlag, New York, 1996.

[29] H. Triebel, Theory of Function Spaces, Birkhäuser Verlag, Boston 1983.

[30] H. Triebel, Interpolation Theory, Function Spaces, Differential Operators, North-Holland, Amsterdam 1978 (2-nd edition, Johann Ambrosius Barth Verlag, Heidelberg-Leipzig 1995). 